\title{
Planning for the next influenza H1N1 season: a modelling study
}

Fabrice Carrat ${ }^{1,2,35^{*}}$, Camille Pelat ${ }^{1,2}$, Daniel Levy-Bruhll ${ }^{4}$ Isabelle Bonmarin ${ }^{4}$, Nathanael Lapidus ${ }^{1,2}$

\begin{abstract}
Background: The level of herd immunity before and after the first 2009 pandemic season is not precisely known, and predicting the shape of the next pandemic H1N1 season is a difficult challenge.

Methods: This was a modelling study based on data on medical visits for influenza-like illness collected by the French General Practitioner Sentinel network, as well as pandemic H1N1 vaccination coverage rates, and an individual-centred model devoted to influenza. We estimated infection attack rates during the first 2009 pandemic H1N1 season in France, and the rates of pre- and post-exposure immunity. We then simulated various scenarios in which a pandemic influenza H1N1 virus would be reintroduced into a population with varying levels of protective cross-immunity, and considered the impact of extending influenza vaccination.
\end{abstract}

Results: During the first pandemic season in France, the proportion of infected persons was 18.1\% overall, 38.3\% among children, $14.8 \%$ among younger adults and 1.6\% among the elderly. The rates of pre-exposure immunity required to fit data collected during the first pandemic season were $36 \%$ in younger adults and $85 \%$ in the elderly. We estimated that the rate of post-exposure immunity was $57.3 \%$ (95\% Confidence Interval (95\%Cl) 49.6\%-65.0\%) overall, $44.6 \%(95 \% \mathrm{Cl} 35.5 \%-53.6 \%)$ in children, $53.8 \%(95 \% \mathrm{Cl} 44.5 \%-63.1 \%)$ in younger adults, and $87.4 \%(95 \% \mathrm{Cl}$ $82.0 \%-92.8 \%)$ in the elderly.

The shape of a second season would depend on the degree of persistent protective cross-immunity to descendants of the $2009 \mathrm{H} 1 \mathrm{~N} 1$ viruses. A cross-protection rate of $70 \%$ would imply that only a small proportion of the population would be affected. With a cross-protection rate of 50\%, the second season would have a disease burden similar to the first, while vaccination of $50 \%$ of the entire population, in addition to the population vaccinated during the first pandemic season, would halve this burden. With a cross-protection rate of 30\%, the second season could be more substantial, and vaccination would not provide a significant benefit.

Conclusions: These model-based findings should help to prepare for a second pandemic season, and highlight the need for studies of the different components of immune protection.

\section{Background}

On 11 June 2009, WHO announced the first influenza pandemic of the 21st century, following the emergence of a new influenza $\mathrm{A} / \mathrm{H} 1 \mathrm{~N} 1$ virus in Mexico and its rapid worldwide spread. By March 2010 most countries had experienced a season of pandemic influenza H1N1, with one or occasionally two peaks. Surveillance reports showed that the burden of illness during this first season did not differ much from that of recent seasonal influenza epidemics [1-4], apart from a risk of unusually severe pneumonia in young people[5-15]. However, the

\footnotetext{
* Correspondence: fabrice.carrat@free.fr

${ }^{1}$ Université Pierre et Marie Curie - Paris 6, UMR-S 707, Paris, F-75012, France Full list of author information is available at the end of the article
}

true infection rates in the general population remain poorly documented.

Two parameters are of critical importance for interpreting surveillance data collected during this first pandemic season: first, the proportion of the population that was susceptible to infection before the 2009 pandemic influenza A/H1N1 virus (hereafter referred to as 2009 H1N1) started to circulate; and second, the rate of asymptomatic or paucisymptomatic infection. Several studies suggest that a substantial proportion of the population, and particularly the elderly, had pre-existing cross-reactive antibodies against 2009 H1N1 [16-18], and that asymptomatic or paucisymptomatic infection was relatively frequent $[18,19]$. Consequently, the level of 
pre- or post-exposure immunity is difficult to estimate, hindering attempts to predict the shape of a subsequent pandemic H1N1 season.

Here we estimate the infection attack rates during the first 2009 pandemic H1N1 season in France and attempt to predict the shape of a second season of pandemic H1N1 by using an individual-centred model [20]. We estimated the first season infection attack rates in three age groups: children, adults under 65 , and the elderly, based on different postulates for the proportion of asymptomatic or paucisymptomatic infection. We then fitted the model to these attack rates according to preexposure cross-immunity and vaccine uptake, in order to derive the size of the immune population after the first pandemic season. Finally, we envisaged various scenarios in which pandemic influenza H1N1 viruses escaping immunity (due to viral evolution and loss of immunity) would be reintroduced, and evaluated the likely impact of extending $2009 \mathrm{H} 1 \mathrm{~N} 1$ influenza vaccination.

\section{Methods}

\section{Estimates of the infected population from national surveillance data}

We used data from the French General Practitioner (GP) Sentinel network [21]. The network is a continuous epidemiological surveillance system based on voluntary GPs and operating since 1984 in France. Sentinel GPs report cases of influenza-like illness (ILI), defined as abrupt-onset fever above $39^{\circ} \mathrm{C}$ accompanied by respiratory signs and symptoms and myalgia or stiffness. Weekly national ILI incidence was estimated from the average number of ILI cases reported by GPs participating in surveillance during a week, multiplied by the ratio of all French GPs to participating sentinel GPs [22]. Surveillance criteria and procedures were not modified during the first pandemic season.

Three age groups were considered: children ( $0-18$ years), younger adults ( $<65$ years), and the elderly ( $\geq 65$ years). In order to estimate the total size of the infected population, we took into account the fact that some cases of ILI might have been caused by other pathogens (poorly specific case definition), and that not all cases of influenza virus infection would result in ILI corresponding to the case definition (lack of sensitivity). The latter cases would include asymptomatic and paucisymptomatic infection. We also took into account the fact that not all patients with typical ILI seek medical advice (figure 1).

To overcome the poor specificity of the clinical case definition, we calculated the excess of GP consultations by children and adults under 65 , relative to baseline rates, using seasonal regression models fitted to historical data since 1985, as described elsewhere [23]. The seasonal regression model was used to fit all-ages weekly incidence data between 1985 to 2010, defining the first

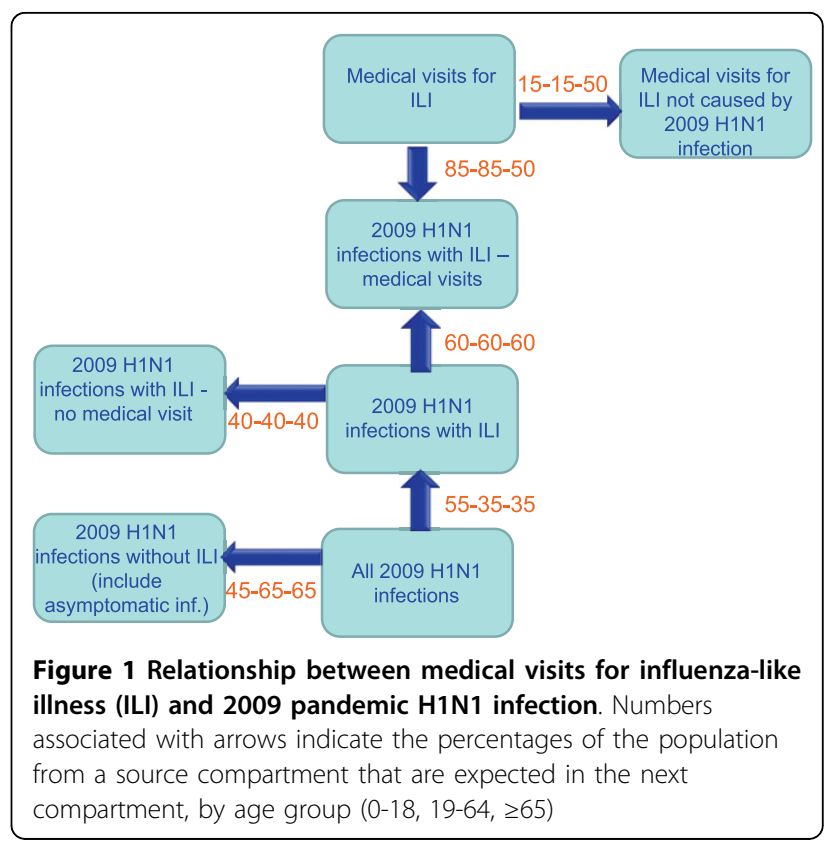

French pandemic season as the period during which the incidence of medical visits for ILI exceeded the upper $90 \%$ limit of the predicted incidence for at least two consecutive weeks. The excess attributed to 2009 H1N1 was calculated in the same way, using a separate model for each age group, by summing the weekly differences between the observed and predicted incidence rates during the first pandemic season. We used a different method for elderly subjects, among whom the seasonality of medical visits for ILI was less clear-cut. We assumed that $50 \%$ of medical visits for ILI in this age group during the first pandemic season were associated with 2009 H1N1 infection, while rates of $0-100 \%$ were used to calculate confidence intervals. In children and younger adults, excess medical visits attributed to 2009 H1N1 represented, on average, $85 \%$ of all medical visits for ILI during the first pandemic season.

The proportion of patients with ILI who did not seek medical advice was estimated from a monthly telephone survey, conducted since May 2009, of a representative sample of 800 members of the general population (unpublished data). Overall, approximately $40 \%$ of persons who reported having typical 'flu-like symptoms did not consult a GP, which is consistent with the results of a prospective follow-up survey of 817 household contacts of index cases with seasonal influenza virus infection (43\%) [24]. Finally, we used values from a meta-analysis of experimental human influenza challenge studies showing that approximately $65 \%$ of infected volunteers did not develop typical 'flu-like illness (33\% did not have symptoms, $32 \%$ did not have fever), and who would not thus have matched our case definition [25]. The proportion of 
infections not matching the ILI definition was assumed to be lower in children (45\%), who are considered more likely than adults to develop fever [26].

Population data and demographic parameters were obtained from national censuses [27]. In France, pandemic influenza vaccination started in November 2009, initially in groups at risk of complications. Influenza vaccine coverage rates were obtained from the French national security database. Adjuvanted vaccines were used in the vast majority of cases. At the end of first pandemic season the vaccine coverage rates were $12.6 \%$ in children, $6.8 \%$ in younger adults, and $6.7 \%$ in the elderly, most subjects having received a single injection. Confidence limits for proportions were calculated with the delta method.

\section{Fitting the first pandemic $\mathrm{H} 1 \mathrm{~N} 1$ season}

We used an individual-centred model, which permits rich parameterization of the simulated population [20]. The model included detailed descriptions of healthcare use and interventions aiming at controlling influenza. It also included demographic characteristics and household sizes, and simulated the spread of influenza through the use of randomly generated graphs. The random graphs were a mixture of bidirectional graphs, comprising fullyconnected graphs for describing contact pattern within the household, and Barabasi-Albert scale free graphs [28] for describing other social contacts. The networks exhibit a substantial level of clustering meaning that two simulated individuals have an increased chance to be contacts of each other given that they share a common network contact. The connectivity of the simulated network followed a power law distribution, with some individuals having a large number of contacts which allows generation of superspreading events

The mean number of contacts per subject (the connectivity of the network) was 13.9 overall (standard deviation $\mathrm{SD}=0.4), 15.3(\mathrm{SD}=0.06)$ for children, 14.6 $(\mathrm{SD}=0.59)$ for younger adults, and $5.1(\mathrm{SD}=0.16)$ for the elderly, in keeping with the results of recent large surveys $[29,30]$. New networks were generated at each simulation.

We made the following assumptions:

- We used realistic modelling of infectivity based on experimental infection viral shedding data [25]. Rather than assuming that infectivity was constant, we modelled it as a function depending on the time elapsed from infection [31]. We assumed the kinetics of infectivity had a gamma density function form (shape parameter $=5.2$, scale parameter $=1$ ), with an offset of 0.5 day (a latent period) and the function was truncated at ten days. Infectivity did not depend on age [32] and peaked at 2.1 days, with a calculated generation time of 2.6 days [33-35]. Infectivity was scaled during the fitting process to adjust the observed data. The resulting probability of transmission during a hypothetical meeting lasting throughout the infective period between a susceptible and a single infected individual was $40 \%$.

- Children were fully susceptible to infection, and unknown proportions of the younger adult and elderly populations (to be calculated) were immune to 2009 H1N1 before it started to circulate. We assumed that these immune subjects could not be infected, irrespective of the number of contacts with infectious persons ("all-or-nothing" protection) [36].

- Subjects with asymptomatic infection were half as infective as other subjects, and, among subjects who consulted a GP, $40 \%$ did so the first day after symptom onset, 30\% the second day, and 30\% later than the second day [24].

- We postulated that $70 \%$ of individuals who consulted a GP would remain confined to home for five days, as recommended [37].

- We assumed that $50 \%$ of patients who visited a doctor within two days of symptom onset received antiviral therapy. We also assumed that antiviral treatment reduced an individual's infectiousness by $28 \%$ [38], and their risk of severe influenza by $80 \%[5,7,12,13]$. Antiviral prophylaxis was not considered, as it was not recommended in France during the first H1N1 season.

- For consistency with observed vaccine coverage rates, we assumed that vaccination started 4 weeks after the outset of the epidemic and increased linearly over the next 7 weeks. We assumed that influenza vaccination was $80 \%$ protective against infection and illness, irrespective of age, starting 15 days after vaccination. Vaccination was administered irrespective of the individual's history of exposure or immunity to influenza viruses.

The model was calibrated by varying the proportion of younger adults and elderly subjects with pre-exposure immunity to fit the excess rates of medical visits attributed to 2009 H1N1 in the relevant age group. For each set of parameters we ran 400 simulations, starting with a single infectious individual at the first day of the first French pandemic season. We classified as "outbreaks" situations in which more than 5 per 1000 subjects were infected [20]. Goodness-of-fit was optimized by minimising the difference by age group between the observed and average rates in simulated outbreaks. The size of the post-exposure immunized population was estimated in each of the three age groups as the proportion of individuals who were infected during the first pandemic season or who were immunized naturally or by vaccination prior to the first pandemic season. Confidence limits for the rates of post-exposure immunity were calculated, assuming that the rate of pre-exposure immunity could vary between $+5 \%$ and $-5 \%$ of the values obtained in the fitted model. 
We also simulated a scenario in which the entire population was susceptible before introducing infectious individuals, all other parameters being equal, in order to examine how a total lack of pre-exposure immunity might influence the first pandemic season.

We calculated the effective reproductive number by simulating the first generation of secondary cases after introducing a single infectious subject, as described elsewhere [20]. We also calculated a basic reproductive number by setting all parameters related to healthcare use (treatment, isolation, etc.), and the size of the preexposure immune population, to zero.

Scenarios: reintroduction of pandemic H1N1 viruses with modified antigenic properties, varying levels of crossprotection, and different vaccination strategies

We simulated reintroduction of individuals infected by a pandemic $\mathrm{H} 1 \mathrm{~N} 1$ virus exhibiting modified antigenic properties (due to antigenic drift for example), at a rate of $2 / 1000$, in a population in which individuals who were immune after the first season had varying levels of persistent protective cross-immunity against the new virus [39]. For these individuals, the chance of being infected during contact with an infected individual was reduced by $90 \%$ to $30 \%$. All other parameters (transmission parameters, pathogenicity, contact networks and healthcare use) remained identical to those used to adjust the first epidemic curve. We completed each scenario by postulating that $10 \%$ to $50 \%$ of the entire population (or children) who were not vaccinated during the first pandemic season would receive the $2009 \mathrm{H} 1 \mathrm{~N} 1$ vaccine before the reintroduction of infectious individuals. Vaccine effectiveness was reduced in proportion to the postulated cross-protection (vaccine effectiveness $=80 \% \mathrm{x}$ crossprotection), as the mechanism underlying the loss of naturally acquired immunity would also concern vaccination-induced immunity, as vaccines being prepared for the 2010-2011 season cover 2009 H1N1 [40].

\section{Results}

\section{Estimates of the infected population from national} surveillance data

The first pandemic season in France lasted 16 weeks, from 7 September 2009 to 27 December 2009. The incidence of medical visits for ILI increased moderately and remained at a stable low level during the first 6 weeks, then increased more sharply and peaked between 6 and 12 December.

During the course of the first pandemic season, we estimated that the proportions of the population who consulted a GP for ILI were $4.86 \%$ overall $(95 \% \mathrm{CI}$ $3.62 \%-6.11 \%$ ), $12.7 \%$ among children (95\%CI $11.3 \%$ $14.0 \%), 3.11 \%$ (95\%CI $1.67 \%-4.56 \%)$ among younger adults, and $0.34 \%$ among the elderly (95\%CI $0-0.68 \%$ ) (figure 2). The estimated proportion of the population infected by pandemic H1N1 was $18.1 \%$ overall $(95 \% \mathrm{CI}$ $12.2 \%-23.9 \%)$, 38.3\% (95\%CI 30.8\%-45.9\%) among children, $14.8 \%$ (95\%CI 7.01\%-22.6\%) among younger adults, and $1.62 \%$ (95\% CI 0\%-3.60\%) among the elderly.

\section{Fitting the first pandemic $\mathrm{H} 1 \mathrm{~N} 1$ season}

The model was fitted to the excess rates of medical visits attributed to $2009 \mathrm{H} 1 \mathrm{~N} 1$ by setting the pre-exposure immune populations to $36 \%$ among younger adults and $85 \%$ among the elderly. The simulated proportion of infected persons was $18.2 \%$ overall (InterQuartile Range (IQR) $17.2 \%-20.7 \%$ ), $39.3 \%$ in children (IQR $37.3 \%$ $44.0 \%$ ), $14.8 \%$ in younger adults (IQR $13.0 \%-17.1 \%$ ) and $1.49 \%$ in the elderly (IQR $1.21 \%-1.76 \%$ ). The simulated outbreaks lasted an average of 13.1 weeks (IQR 11-14 weeks), $10 \%$ of outbreaks exceeding 16 weeks.

The post-exposure immune population represented $57.3 \%$ overall (95\%CI $49.6 \%-65.0 \%$ ), $44.6 \%$ (95\% CI $35.5 \%-53.6 \%)$ in children, $53.8 \%$ (95\%CI $44.5 \%-63.1 \%)$ in younger adults, $87.4 \%(82.0 \%-92.8 \%)$ in the elderly.

Postulating no pre-exposure immunity in younger adults and elderly persons, the simulated proportions of infected persons would be $47.9 \%$ overall (IQR $46.2 \%$ $49.7 \%$ ), $64.2 \%$ in children (IQR 62.6\%-65.4\%), $47.6 \%$ in younger adults (IQR 45.4\%-49.7\%) and $26.7 \%$ among the elderly (IQR 25.2\%-27.9\%). An estimated 11.9\% (11.4\%$12.4 \%)$ of the total population would consult a GP for ILI caused by 2009 H1N1.

The effective reproductive number was 1.03 and the basic reproductive number 1.54 (figure 3 ). An average of 1.57 persons were infected ( 1.11 children, 0.45 younger adults and 0.01 elderly persons) when the index patient

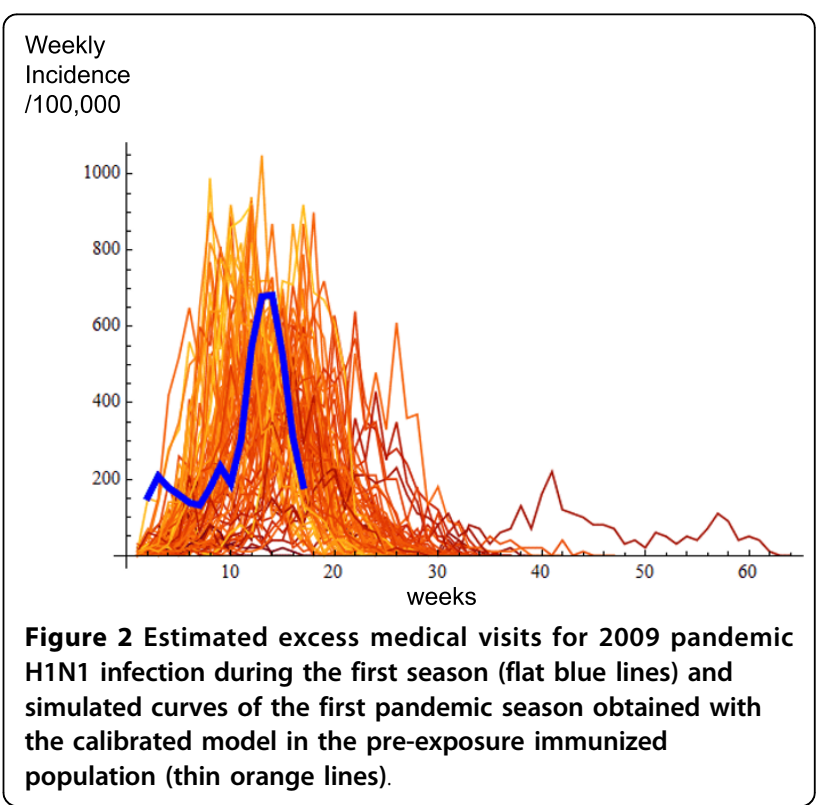




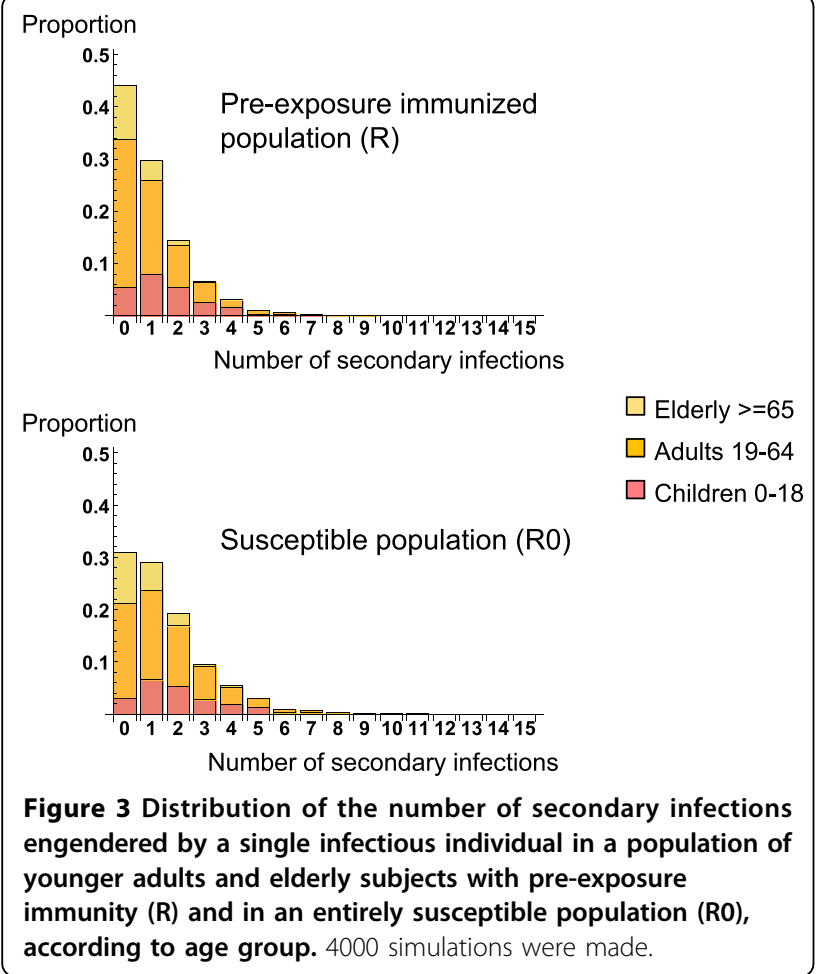

was a child, 0.96 ( 0.23 children, 0.72 younger adults, 0.01 elderly) when the index patient was a younger adult, and 0.43 ( 0.14 children, 0.25 younger adults, 0.04 elderly persons) when the index patient was an elderly person.

\section{Scenarios: reintroduction of pandemic H1N1 viruses with modified antigenic properties, varying levels of cross- protection, and different vaccination strategies}

As shown in Table 1, persistent cross-protection above $70 \%$ would be necessary to markedly limit the size of a second pandemic season due to a virus with different antigenic properties. Cross-protection of $50 \%$ would imply attack rates of $23.1 \%$ overall, and rates notably higher in younger adults and the elderly than during the first pandemic season. Influenza vaccination of 30 to $50 \%$ of the population, in addition to the population vaccinated during the first pandemic season, would halve the attack rates, provided that cross-protection was not below $50 \%$. With cross-protection below $30 \%$, vaccination of $50 \%$ of the population would have little impact. Vaccination of children alone would limit the burden of influenza in all age groups if cross-protection was between $50 \%$ and $70 \%$ and the vaccine coverage rate exceeded $50 \%$; in contrast, this measure would have little impact in case of cross-protection greater than $70 \%$; and would be barely effective in case of crossprotection below $50 \%$.

\section{Discussion}

Between $12 \%$ and $24 \%$ of the French population were infected by the $2009 \mathrm{H} 1 \mathrm{~N} 1$ pandemic virus during autumn and winter 2009. The cumulative incidence rate was much higher in children (38.3\%) than in younger adults (14.8\%) and the elderly (1.62\%).

Here, using a modelling approach parameterized with the best available epidemiological data for the French 2009 H1N1 season, and taking into account the behaviour of patients with ILI and implementation of control measures, we show that substantial pre-exposure immunity to 2009 $\mathrm{H} 1 \mathrm{~N} 1-36 \%$ in younger adults and $85 \%$ in the elderly would be necessary to fit the observed incidence rates.

The likelihood of a major second 2009 H1N1 season would depend on the degree of persistent protective crossimmunity against new 2009 H1N1 variants. Assuming cross-protection of $70 \%$ among people infected during the first $2009 \mathrm{H} 1 \mathrm{~N} 1$ season and among those who were already protected, a second season would affect only a small proportion of the population. With cross-protection of $50 \%$, the second season would have a disease burden similar to that of the first season, and vaccination of $50 \%$ of the entire population, in addition to the population vaccinated during the first pandemic season, would more than halve this burden. With cross-protection of $30 \%$, the second season could be substantial, even if vaccine coverage increased by $50 \%$.

Our estimates of the cumulative incidence rates of 2009 H1N1 infection during the first pandemic season are slightly higher than those obtained in a serological survey conducted in London and the West Midlands, which suggested that $21.3 \%$ of < 5 -year-olds, $42.0 \%$ of 5 - to 14 year-olds, $20.6 \%$ of 15 - to 24 -year-olds, $6.2 \%$ of 25 - to 44 year-olds and only $0.9 \%$ of the elderly were infected [18]. These incidence rates were based on differences in the proportion of samples with haemagglutination inhibition titres of 1:32 or higher between 2008 and September 2009, and did not take into account 2009 H1N1 infections occurring in late October-early November 2009. Also, 11\% of individuals who had PCR-confirmed 2009 H1N1 infection had not seroconverted after 21 days, indicating that 2009 infection rates were underestimated. In another study, post-exposure $2009 \mathrm{H} 1 \mathrm{~N} 1$ seroprevalence rates were $45 \%$ in children aged 10 to 19 years, $14 \%$ to $22 \%$ in adults under 60 , and $5 \%$ to $26 \%$ in adults aged 60 to 89 [41], giving an overall rate of $21.5 \%$. Our findings are compatible with these data. Interestingly, in this latter study, several findings pointed to protective crossimmunity due to exposure to previous H1N1 viruses. In the elderly population, antibodies to the $1918 \mathrm{H} 1 \mathrm{~N} 1$ virus were found in $48 \%$ to $57 \%$ of cases, while antibodies to the $1957 \mathrm{H} 1 \mathrm{~N} 1$ virus (a descendant of $1918 \mathrm{H} 1 \mathrm{~N} 1$ ) were found in $37 \%$ to $58 \%$ of adults aged 40 to 59 . Cross- 
Table 1 Simulated infection rates during a second pandemic influenza H1N1 season according to persistent cross-protection and increasing vaccine coverage Increased vaccine coverage

\begin{tabular}{|c|c|c|c|c|c|c|c|}
\hline \multirow[b]{3}{*}{ Persistent protective cross-immunity (\%) } & \multirow[b]{3}{*}{ No vaccination } & \multicolumn{6}{|c|}{$\begin{array}{l}\text { Increased vaccine coverage } \\
(\%)\end{array}$} \\
\hline & & \multicolumn{3}{|c|}{ All ages } & \multicolumn{3}{|c|}{$0-18$ yrs } \\
\hline & & 10 & 30 & 50 & 30 & 50 & 70 \\
\hline 90 & $\begin{array}{l}2.35(0.66-2.85) \\
1.76(0.48-2.49) \\
0.29(0.12-0.36) \\
\mathbf{1 . 6 5}(\mathbf{0 . 5 1 - 2 . 3 1 )}\end{array}$ & $\begin{array}{l}1.42(0.53-1.89) \\
1.11(0.31-1.35) \\
0.16(0.06-0.18) \\
\mathbf{1 . 0 2}(\mathbf{0 . 3 5}-1.35)\end{array}$ & $\begin{array}{l}0.68(0.26-0.88) \\
0.59(0.20-0.72) \\
0.10(0.00-0.12) \\
\mathbf{0 . 5 3}(\mathbf{0 . 2 3 - 0 . 6 6 )}\end{array}$ & $\begin{array}{l}0.30(0.13-0.39) \\
0.26(0.12-0.30) \\
0.05(0.00-0.06) \\
\mathbf{0 . 2 3}(\mathbf{0 . 1 3 - 0 . 2 6 )}\end{array}$ & $\begin{array}{l}1.05(0.39-1.58) \\
1.40(0.33-1.86) \\
0.21(0.06-0.30) \\
\mathbf{1 . 1 2}(\mathbf{0 . 3 4 - 1 . 4 4 )}\end{array}$ & $\begin{array}{l}0.65(0.26-0.88) \\
1.21(0.33-1.70) \\
0.14(0.00-0.18) \\
\mathbf{0 . 9 1}(\mathbf{0 . 2 7 - 1 . 2 1 )}\end{array}$ & $\begin{array}{l}0.40(0.13-0.57) \\
1.26(0.31-1.94) \\
0.16(0.06-0.24) \\
\mathbf{0 . 8 8}(\mathbf{0 . 2 4}-\mathbf{1 . 3 2})\end{array}$ \\
\hline 70 & $\begin{array}{l}14.0(11.2-18.4) \\
10.3(7.33-14.3) \\
2.65(1.94-3.63) \\
\mathbf{9 . 9 1}(\mathbf{7 . 2 9 - 1 3 . 9 )}\end{array}$ & $\begin{array}{l}8.44(2.68-13.8) \\
6.17(1.66-10.4) \\
1.56(0.55-2.48) \\
\mathbf{5 . 9 2}(\mathbf{1 . 7 1 - 9 . 8 2 )}\end{array}$ & $\begin{array}{l}3.51(0.83-5.04) \\
2.81(0.59-3.94) \\
0.66(0.18-0.97) \\
\mathbf{2 . 6 1}(\mathbf{0 . 5 9 - 3 . 6 5 )}\end{array}$ & $\begin{array}{l}1.24(0.48-1.62) \\
1.13(0.30-1.20) \\
0.29(0.06-0.36) \\
\mathbf{1 . 0 2}(\mathbf{0 . 3 2 - 1 . 1 8 )}\end{array}$ & $\begin{array}{l}6.20(1.54-9.96) \\
6.05(1.63-10.2) \\
1.45(0.42-2.42) \\
\mathbf{5 . 3 2}(\mathbf{1 . 3 7 - 8 . 7 8 )}\end{array}$ & $\begin{array}{l}4.76(1.36-7.11) \\
6.56(1.45-10.2) \\
1.46(0.42-2.18) \\
\mathbf{5 . 3 1}(\mathbf{1 . 3 1 - 8 . 3 9 )}\end{array}$ & $\begin{array}{l}2.73(0.66-4.30) \\
4.61(0.69-8.14) \\
1.06(0.18-1.76) \\
\mathbf{3 . 6 0}(\mathbf{0 . 6 3 - 6 . 2 0 )}\end{array}$ \\
\hline 50 & $\begin{array}{l}33.2(30.9-36.3) \\
23.1(20.7-25.8) \\
8.85(7.70-10.1) \\
\mathbf{2 3 . 1}(\mathbf{2 0 . 7 - 2 5 . 2 )} \\
\end{array}$ & $\begin{array}{l}29.4(26.3-33.3) \\
21.3(18.4-24.3) \\
7.99(7.09-9.03) \\
\mathbf{2 0 . 9}(\mathbf{1 8 . 6 - 2 3 . 5 )} \\
\end{array}$ & $\begin{array}{l}20.2(18.1-25.4) \\
15.5(12.0-20.4) \\
5.76(4.73-7.27) \\
\mathbf{1 5 . 0}(\mathbf{1 2 . 4 - 1 9 . 2 )} \\
\end{array}$ & $\begin{array}{l}14.0(9.91-19.4) \\
11.6(6.95-15.5) \\
4.16(2.55-5.82) \\
\mathbf{1 0 . 9}(\mathbf{0 . 6 9 - 1 5 . 2 )} \\
\end{array}$ & $\begin{array}{l}25.6(23.8-28.4) \\
20.9(18.4-24.2) \\
7.82(6.91-8.97) \\
\mathbf{1 9 . 8}(\mathbf{1 8 . 0 - 2 2 . 8 )} \\
\end{array}$ & $\begin{array}{l}21.4(19.6-24.6) \\
19.5(16.4-22.8) \\
7.13(6.42-8.18) \\
\mathbf{1 7 . 9}(\mathbf{1 5 . 6 - 2 0 . 6 )} \\
\end{array}$ & $\begin{array}{l}17.3(14.9-21.8) \\
18.7(16.0-23.7) \\
6.47(5.33-8.24) \\
\mathbf{1 6 . 4}(\mathbf{1 3 . 7 - 2 0 . 7 )}\end{array}$ \\
\hline 30 & $\begin{array}{l}49.7(48.3-51.4) \\
35.2(32.5-37.8) \\
16.6(15.2-17.9) \\
\mathbf{3 5 . 4}(\mathbf{3 3 . 2}-\mathbf{3 7 . 5 )}\end{array}$ & $\begin{array}{l}46.5(44.9-48.9) \\
34.0(31.6-36.3) \\
15.7(14.4-17.0) \\
\mathbf{3 3 . 8}(\mathbf{3 2 . 3 - 3 6 . 2 )}\end{array}$ & $\begin{array}{l}43.6(41.3-45.9) \\
31.7(28.9-33.7) \\
14.7(13.6-15.8) \\
\mathbf{3 1 . 6}(\mathbf{2 9 . 1 - 3 3 . 4 )}\end{array}$ & $\begin{array}{l}39.7(37.5-42.5) \\
29.1(27.2-31.6) \\
13.7(12.8-14.5) \\
\mathbf{2 9 . 0}(\mathbf{2 7 . 3 - 3 1 . 1 )}\end{array}$ & $\begin{array}{l}45.1(43.0-47.1) \\
34.6(32.4-36.9) \\
15.9(14.9-16.9) \\
\mathbf{3 3 . 9}(\mathbf{3 1 . 9 - 3 5 . 8 )}\end{array}$ & $\begin{array}{l}41.7(40.2-44.0) \\
32.6(30.3-35.3) \\
15.1(13.9-16.6) \\
\mathbf{3 1 . 8}(\mathbf{3 0 . 2 - 3 4 . 0 )}\end{array}$ & $\begin{array}{l}39.8(37.7-42.4) \\
32.8(30.0-36.0) \\
15.2(14.1-16.4) \\
\mathbf{3 1 . 5}(\mathbf{2 9 . 2 - 3 4 . 2 )}\end{array}$ \\
\hline
\end{tabular}

In each cell, mean infection rates per 100, in children (0-18 years), adults under 65 years and elderly (standard style) and total population (bold style) are given with their interquartile ranges (in parentheses). 
reactivity between antibodies elicited by $\mathrm{H} 1 \mathrm{~N} 1$ viruses circulating up to 1957 is further supported by the antigenic similarity [42] and reported cross-neutralization [43] between these viruses and 2009 H1N1. Together, these findings help to explain the low attack rates observed in the adult and elderly populations. However, the precise level of pre- or post-exposure immunity to 2009 H1N1 is difficult to evaluate. Serological analyses only measure adaptive immunity, failing to quantify cellular and innate immunity. Moreover, the protection associated with haemagglutination inhibition or neutralization antibody titres is not known for 2009 H1N1.

We found evidence of elevated levels of pre-exposure immunity in the French adult and elderly populations, yielding a reproductive number slightly higher than 1 . Such a low reproductive number is supported by the highly variable patterns of the first 2009 H1N1 seasons across various countries, with one or two peaks, a lack of spatial synchrony, and moderate clinical attack rates [44]. We estimated the reproductive number on our data using the early epidemic growth rate (fitted on incidence data from one week before to two weeks after the epidemic onset, using the method described in [45], with a generation time of 2.6 days and a ratio of infectious period to the generation time of 0.61 ). The value obtained (1.18) was close to our estimate and lower than the average estimate of 1.3 during seasonal influenza epidemics in France [46]. Estimates of the final size of the infected population, taking into account relative susceptibilities in different age groups also closely matched our estimates (17.5\%) [47]. However, our reproductive number was substantially lower than those reported in the US or Mexico for the first pandemic season [33,34]. This apparent discrepancy may be the consequence of estimations in different time, settings or countries but may also be explained by our modelling framework. In scale-free networks of finite size, the heterogeneity of scale-free connectivity patterns favors epidemic spreading by lowering the epidemic threshold [48]. In contrast with homogeneous network, the scale-free network allows epidemic spreading for a low average number of infections produced by an infected individual [49].

Our calculations of pre-exposure protective crossimmunity may have been influenced by assumptions concerning the proportion of infected individuals who did not develop ILI. It has been estimated that the proportion of all infected subjects who visited their GP in France was $19.6 \%$ among pregnant women [19], a figure in line with our postulate of $21 \%$ in the 19 - to 64 -year age group. When we postulated lower proportions of individuals with ILI among those infected with 2009 H1N1 [18], the cumulative incidence rates of infection increased and the level of pre-exposure immunity necessary to fit the epidemic curve therefore decreased. In this case, transmission parameters would also increase, and our estimates of the post-exposure immunized population would not be markedly affected.

There are few reports on cross-protection between successive pandemic or seasonal influenza seasons. The cross-protective effect was estimated to range from $35 \%$ to $94 \%$ for clinical illness between the spring and summer waves and the autumn wave during the 1918 pandemic [50], while no evidence of cross-protection was found between the autumn wave and a third winter wave. As the 1918 pandemic H1N1 virus was antigenically close to $2009 \mathrm{H} 1 \mathrm{~N} 1$, a strong decline in protection could occur if the same situation is repeated. Genetic characterization of 2009 H1N1 has already identified different evolving clades and complex spatio-temporal dynamics [51], and significant drift before the next season is likely.

Mass vaccination with the 2009 H1N1 influenza vaccine would be effective only within a limited range of crossprotection against a re-emerging H1N1 strain. Even if cross-reactive antibodies might have been elicited by adjuvanted influenza vaccination (used in late 2009/early 2010 in France), there is no evidence that adjuvanted vaccines provide superior cross-protection than naturally-acquired infection against drifted strains, and we therefore applied the same reasoning to individuals who were naturally immunized and those who were vaccinated.

The next pandemic season, if it occurs, could affect more adults and elderly subjects than the first. This was the case in past pandemics [52] and has been carefully analyzed in a network-based modelling study, in which a shift to older age between the first and subsequent seasons was predicted [53]. As the case-fatality ratio was higher in the adult and elderly populations than in children $[15,54]$, the mortality burden of a subsequent H1N1 pandemic season due to a virus with unchanged pathogenicity could be higher than during the first season.

\section{Conclusions}

Pre-exposure immunity to $2009 \mathrm{H} 1 \mathrm{~N} 1$ influenza virus was higher than anticipated in French adults and elderly people. A sustained high level of cross-protection against descendants of the $2009 \mathrm{H} 1 \mathrm{~N} 1$ virus would be necessary to avoid a second significant 2009 H1N1 season. Extending influenza vaccination across all age groups would be effective if cross-protection against descendants of the $2009 \mathrm{H} 1 \mathrm{~N} 1$ virus ranged between $30 \%$ to $70 \%$, but would not provide a significant benefit in other situations. This study therefore highlights the need for comprehensive studies of the different components of immune protection, and the need to maintain worldwide virological and ILI surveillance for early detection of antigenic drift. 


\section{Abbreviations}

WHO: World Health Organization; ILI: Influenza-Like IIIness; GP: General Practitioner; SD: Standard Deviation; 95\%Cl: 95\% Confidence Interval; IQR: InterQuartile Range.

\section{Acknowledgements}

We thank Alain-Jacques Valleron (Professor, public health, Saint-Antoine hospital) for his advice. This work was made possible thanks to the 2300 SGPs who, since November 1st, 1984 sent more than 300000 case descriptions of ILI that constituted the ongoing influenza database analyzed herein, and to the 'Sentinelles' research team, particularly P. Garnerin, A. Flahault, T. Hanslik, and T. Blanchon, PY. Boelle, F. Carrat, G. Gropetis, C. Pelat, C. Turbelin.

The study was supported by Université Pierre et marie Curie-Paris6 and INSERM and by Institut de Veille Sanitaire. The study funders have no role in the study design; in the collection, analysis, and interpretation of data; in the writing of the report; and in the decision to submit the article for publication. Researchers were independent from funders.

All authors have full access to all of the data (including statistical reports and tables) in the study and can take responsibility for the integrity of the data and the accuracy of the data analysis.

\section{Author details}

${ }^{1}$ Université Pierre et Marie Curie - Paris 6, UMR-S 707, Paris, F-75012, France. ${ }^{2}$ Inserm U707, Paris, F-75012, France. ${ }^{3}$ Assistance Publique Hôpitaux de Paris, Hôpital Saint Antoine, Paris, F-75012, France. ${ }^{4}$ Département des maladies infectieuses, Institut de Veille Sanitaire, Saint-Maurice; 94415, France. ${ }^{5} \mathrm{~F}$ Carrat, UMR-S 707, Faculté de médecine Saint Antoine, 27 rue Chaligny, 75571 PARIS CEDEX 12, France.

\section{Authors' contributions}

FC conceived the study and designed it with DLB. CP and NL collected data and did experiments for the study. FC, CP, DLB, and NL analyzed the data. FC wrote the first draft of the paper. FC, CP, DLB, IB and NL revised the paper. All authors read and approved the final manuscript.

\section{Competing interests}

FC was a consultant for Novartis and GlaxoSmithKline; CP, DL-B, IB, NL have no relationships with companies that might have an interest in the submitted work in the previous 3 years; their spouses, partners, or children have no financial relationships that may be relevant to the submitted work; and (4) FC, CP, DL-B, IB, NL have no non-financial interests that may be relevant to the submitted work

Received: 4 June 2010 Accepted: 21 October 2010 Published: 21 October 2010

\section{References}

1. Valleron AJ, Guidet B: Real-time comparative monitoring of the A/H1N1 pandemic in France. Clin Microbiol Infect 2010, 16:393-396.

2. Initial epidemiological findings in the European Union following the declaration of pandemic alert level 5 due to influenza $A(H 1 N 1)$. Euro Surveill 2009, 14:pii, 19204.

3. Baker MG, Wilson N, Huang QS, Paine S, Lopez L, Bandaranayake D, Tobias M, Mason K, Mackereth GF, Jacobs M, et al: Pandemic influenza A $(\mathrm{H} 1 \mathrm{~N} 1) \mathrm{v}$ in New Zealand: the experience from April to August 2009. Euro Surveill 2009, 14:pii, 19319.

4. Turbelin C, Pelat C, Boelle PY, Levy-Bruhl D, Carrat F, Blanchon T, Hanslik T: Early estimates of 2009 pandemic influenza $A(\mathrm{H} 1 \mathrm{~N} 1)$ virus activity in general practice in France: incidence of influenza-like illness and age distribution of reported cases. Euro Surveill 2009, 14:pii, 19341.

5. Dominguez-Cherit G, Lapinsky SE, Macias AE, Pinto R, Espinosa-Perez L, de la Torre A, Poblano-Morales M, Baltazar-Torres JA, Bautista E, Martinez A, et al: Critically III patients with 2009 influenza $A(H 1 N 1)$ in Mexico. JAMA 2009, 302:1880-1887.

6. Kumar A, Zarychanski R, Pinto R, Cook DJ, Marshall J, Lacroix J, Stelfox T, Bagshaw S, Choong K, Lamontagne F, et al: Critically ill patients with 2009 influenza A(H1N1) infection in Canada. JAMA 2009, 302:1872-1879,

7. Louie JK, Acosta M, Winter K, Jean C, Gavali S, Schechter R, Vugia D, Harriman K, Matyas B, Glaser CA, et al: Factors associated with death or hospitalization due to pandemic 2009 influenza $A(H 1 N 1)$ infection in California. JAMA 2009, 302:1896-1902

8. Echevarria-Zuno S, Mejia-Arangure JM, Mar-Obeso AJ, Grajales-Muniz C, Robles-Perez E, Gonzalez-Leon M, Ortega-Alvarez MC, Gonzalez-Bonilla C, Rascon-Pacheco RA, Borja-Aburto VH: Infection and death from influenza A H1N1 virus in Mexico: a retrospective analysis. Lancet 2009, 374:2072-2079.

9. Jamieson DJ, Honein MA, Rasmussen SA, Williams JL, Swerdlow DL, Biggerstaff MS, Lindstrom S, Louie JK, Christ CM, Bohm SR, et al: H1N1 2009 influenza virus infection during pregnancy in the USA. Lancet 2009, 374:451-458

10. Cao B, Li XW, Mao Y, Wang J, Lu HZ, Chen YS, Liang ZA, Liang L, Zhang SJ, Zhang $B$, et al: Clinical features of the initial cases of 2009 pandemic influenza A (H1N1) virus infection in China. N Engl J Med 2009, 361:2507-2517.

11. Chowell G, Bertozzi SM, Colchero MA, Lopez-Gatell H, Alpuche-Aranda C, Hernandez M, Miller MA: Severe respiratory disease concurrent with the circulation of H1N1 influenza. N Engl J Med 2009, 361:674-679.

12. Jain S, Kamimoto L, Bramley AM, Schmitz AM, Benoit SR, Louie J, Sugerman DE, Druckenmiller JK, Ritger KA, Chugh R, et al: Hospitalized patients with 2009 H1N1 influenza in the United States, April-June 2009. N Engl J Med 2009, 361:1935-1944.

13. Louie JK, Acosta M, Jamieson DJ, Honein MA: Severe 2009 H1N1 influenza in pregnant and postpartum women in California. N Engl J Med 2010, 362:27-35

14. Perez-Padilla R, de la Rosa-Zamboni D, Ponce de Leon S, Hernandez M, Quinones-Falconi F, Bautista E, Ramirez-Venegas A, Rojas-Serrano J, Ormsby $C E$, Corrales $A$, et al: Pneumonia and respiratory failure from swine-origin influenza A (H1N1) in Mexico. N Engl J Med 2009, 361:680-689.

15. Bautista E, Chotpitayasunondh T, Gao Z, Harper SA, Shaw M, Uyeki TM, Zaki SR, Hayden FG, Hui DS, Kettner JD, et al: Clinical Aspects of Pandemic 2009 Influenza A (H1N1) Virus Infection. N Engl J Med 2010, 362:1708-1719.

16. Serum cross-reactive antibody response to a novel influenza A (H1N1) virus after vaccination with seasonal influenza vaccine. MMWR Morb Mortal Wkly Rep 2009, 58:521-524.

17. Hancock K, Veguilla V, Lu X, Zhong W, Butler EN, Sun H, Liu F, Dong L, DeVos JR, Gargiullo PM, et al: Cross-reactive antibody responses to the 2009 pandemic H1N1 influenza virus. N Engl J Med 2009, 361:1945-1952.

18. Miller $E$, Hoschler $K$, Hardelid $P$, Stanford E, Andrews N, Zambon M: Incidence of 2009 pandemic influenza A H1N1 infection in England: a cross-sectional serological study. Lancet 2010

19. Flahault $A$, de Lamballerie $X$, Hanslik T: Symptomatic infections less frequent with H1N1pdm than with seasonal strains: Antoine Flahault, Xavier de Lamballerie, Camille Pelat, Nicolas Salez, Thomas Hanslik. PLoS Curr Influenza 2009, RRN1140

20. Carrat F, Luong J, Lao H, Salle AV, Lajaunie C, Wackernagel H: A 'smallworld-like' model for comparing interventions aimed at preventing and controlling influenza pandemics. BMC Med 2006, 4:26.

21. Carrat F, Flahault A, Boussard E, Farran N, Dangoumau L, Valleron AJ: Surveillance of influenza-like illness in France. The example of the 1995/ 1996 epidemic. J Epidemiol Community Health 1998, 52(Suppl 1):32S-38S.

22. Garnerin $P$, Saidi $Y$, Valleron AJ: The French Communicable Diseases Computer Network. A seven-year experiment. Ann N Y Acad Sci 1992, 670:29-42.

23. Pelat C, Boelle PY, Cowling BJ, Carrat F, Flahault A, Ansart S, Valleron AJ: Online detection and quantification of epidemics. BMC Med Inform Decis Mak 2007, 7:29.

24. Carrat F, Sahler C, Rogez S, Leruez-Ville M, Freymuth F, Le Gales C, Bungener M, Housset B, Nicolas M, Rouzioux C: Influenza burden of illness: estimates from a national prospective survey of household contacts in France. Arch Intern Med 2002, 162:1842-1848.

25. Carrat F, Vergu E, Ferguson NM, Lemaitre M, Cauchemez S, Leach S, Valleron AJ: Time lines of infection and disease in human influenza: a review of volunteer challenge studies. Am J Epidemiol 2008, 167:775-785

26. Silvennoinen $H$, Peltola $V$, Lehtinen $P$, Vainionpaa R, Heikkinen $T$ : Clinical presentation of influenza in unselected children treated as outpatients. Pediatr Infect Dis J 2009, 28:372-375.

27. Population de la France métropolitaine. [http://www.ined.fr/fr/ pop_chiffres/france/]. 
28. Barabasi AL, Albert R: Emergence of scaling in random networks. Science 1999, 286:509-512.

29. Mossong J, Hens N, Jit M, Beutels P, Auranen K, Mikolajczyk R, Massari M, Salmaso S, Tomba GS, Wallinga J, et al: Social contacts and mixing patterns relevant to the spread of infectious diseases. PLoS Med 2008, 5 e74.

30. Wallinga J, Teunis $P$, Kretzschmar M: Using data on social contacts to estimate age-specific transmission parameters for respiratory-spread infectious agents. Am J Epidemiol 2006, 164:936-944.

31. Ferguson NM, Cummings DA, Fraser C, Cajka JC, Cooley PC, Burke DS: Strategies for mitigating an influenza pandemic. Nature 2006, 442:448-452.

32. Cauchemez S, Donnelly CA, Reed C, Ghani AC, Fraser C, Kent CK, Finelli L, Ferguson NM: Household transmission of 2009 pandemic influenza A (H1N1) virus in the United States. N Engl J Med 2009, 361:2619-2627.

33. White LF, Wallinga J, Finelli L, Reed C, Riley S, Lipsitch M, Pagano M: Estimation of the reproductive number and the serial interval in early phase of the 2009 influenza A/H1N1 pandemic in the USA. Influenza Other Respi Viruses 2009, 3:267-276.

34. Fraser C, Donnelly CA, Cauchemez S, Hanage WP, Van Kerkhove MD, Hollingsworth TD, Griffin J, Baggaley RF, Jenkins HE, Lyons EJ, et al: Pandemic potential of a strain of influenza $A$ (H1N1): early findings. Science 2009, 324:1557-1561.

35. Suess T, Buchholz U, Dupke S, Grunow R, Matthias An der H, Heider A, Biere B, Schweiger B, Haas W, Krause G: Shedding and Transmission of Novel Influenza Virus A/H1N1 Infection in Households-Germany, 2009. Am J Epidemiol 2010

36. Smith $P G$, Rodrigues $L C$, Fine PE: Assessment of the protective efficacy of vaccines against common diseases using case-control and cohort studies. Int J Epidemiol 1984, 13:87-93.

37. Prise en charge médicale des malades atteints de grippe et de leurs contacts en situation de pandémie. [http://www.pandemie-grippale.gouv. fr/IMG/pdf/Fiche_D3-4.pdf].

38. Ferguson NM, Cummings DA, Cauchemez S, Fraser C, Riley S, Meeyai A, lamsirithaworn S, Burke DS: Strategies for containing an emerging influenza pandemic in Southeast Asia. Nature 2005, 437:209-214.

39. Finkenstadt BF, Morton A, Rand DA: Modelling antigenic drift in weekly flu incidence. Stat Med 2005, 24:3447-3461.

40. Carrat F, Flahault A: Influenza vaccine: the challenge of antigenic drift. Vaccine 2007, 25:6852-6862

41. Ross T, Zimmer S, Burke D, Crevar C, Carter D, Stark J, Giles B, Zimmerman R, Ostroff S, Lee B: Seroprevalence Following the Second Wave of Pandemic 2009 H1N1 Influenza. PLoS Curr Influenza 2010, RRN1148.

42. Xu R, Ekiert DC, Krause JC, Hai R, Crowe JE Jr, Wilson IA: Structural Basis of Preexisting Immunity to the 2009 H1N1 Pandemic Influenza Virus. Science 2010.

43. Krause JC, Tumpey TM, Huffman CJ, McGraw PA, Pearce MB, Tsibane T, Hai R, Basler CF, Crowe JE Jr: Naturally occurring human monoclonal antibodies neutralize both 1918 and 2009 pandemic influenza A (H1N1) viruses. J Virol 2010, 84:3127-3130.

44. Viboud C, Bjornstad ON, Smith DL, Simonsen L, Miller MA, Grenfell BT: Synchrony, waves, and spatial hierarchies in the spread of influenza. Science 2006, 312:447-451.

45. Chowell G, Nishiura H, Bettencourt LM: Comparative estimation of the reproduction number for pandemic influenza from daily case notification data. J R Soc Interface 2007, 4:155-166.

46. Chowell G, Miller MA, Viboud C: Seasonal influenza in the United States, France, and Australia: transmission and prospects for control. Epidemiol Infect 2008, 136:852-864.

47. Nishiura H, Chowell G, Safan M, Castillo-Chavez C: Pros and cons of estimating the reproduction number from early epidemic growth rate of influenza A (H1N1) 2009. Theor Biol Med Model 2010, 7:1.

48. Pastor-Satorras R, Vespignani A: Epidemic dynamics in finite size scale-free networks. Phys Rev E Stat Nonlin Soft Matter Phys 2002, 65:035108.

49. May RM, Lloyd AL: Infection dynamics on scale-free networks. Phys Rev E Stat Nonlin Soft Matter Phys 2001, 64:066112

50. Barry JM, Viboud C, Simonsen L: Cross-protection between successive waves of the 1918-1919 influenza pandemic: epidemiological evidence from US Army camps and from Britain. J Infect Dis 2008, 198:1427-1434.
51. Nelson M, Spiro D, Wentworth D, Beck E, Fan J, Ghedin E, Halpin R, Bera J, Hine $E$, Proudfoot $K$, et al: The early diversification of influenza A/ H1N1pdm. PLoS Curr Influenza 2009, RRN1126.

52. Simonsen $L$, Clarke MJ, Schonberger LB, Arden NH, Cox NJ, Fukuda K: Pandemic versus epidemic influenza mortality: a pattern of changing age distribution. $J$ Infect Dis 1998, 178:53-60.

53. Bansal S, Pourbohloul B, Hupert N, Grenfell B, Meyers LA: The shifting demographic landscape of pandemic influenza. PLoS One 2010, 5:e9360.

54. Presanis AM, De Angelis D, Hagy A, Reed C, Riley S, Cooper BS, Finelli L, Biedrzycki P, Lipsitch M: The severity of pandemic H1N1 influenza in the United States, from April to July 2009: a Bayesian analysis. PLoS Med 2009, 6:e1000207.

\section{Pre-publication history}

The pre-publication history for this paper can be accessed here: http://www.biomedcentral.com/1471-2334/10/301/prepub

doi:10.1186/1471-2334-10-301

Cite this article as: Carrat et al:: Planning for the next influenza H1N1 season: a modelling study. BMC Infectious Diseases 2010 10:301.

\section{Submit your next manuscript to BioMed Central and take full advantage of:}

- Convenient online submission

- Thorough peer review

- No space constraints or color figure charges

- Immediate publication on acceptance

- Inclusion in PubMed, CAS, Scopus and Google Scholar

- Research which is freely available for redistribution

Submit your manuscript at www.biomedcentral.com/submit
Biomed Central 